\title{
Three-dimensional modelling of VLF data
}

\author{
David Beamish
}

British Geological Survey, Keyworth, Nottingham NG12 5GG UK

Beamish, D., 1998. Three-dimensional modelling of VLF data. Journal of Applied Geophysics, 39, 63-76.

doi:10.1016/S0926-9851(98)00012-3

Keywords: electromagnetic; modelling; very low frequency

\begin{abstract}
The VLF technique is being increasingly applied to environmental and hydrogeological problems with a growing requirement for quantitative interpretation. One of the main difficulties with the VLF method stems from the directional polarisation of the transmitted field. To clarify VLF data interpretation when subsurface targets are complex and three dimensional, a modelling study was undertaken at VLF frequencies. A main concern is the interpretation of target strike directions when the transmitters used are rotated from principal-mode (i.e., two-dimensional) directions. Results for transmitter strike rotations of between 0 and $75^{\circ}$ of the two principal modes are presented and discussed. For mapping purposes, the invariant apparent resistivity and phase provide important interpretational simplifications. For two-dimensional modelling/inversion, the field distortions introduced by directional complexity remain significant. The results also demonstrate the importance of apparent resistivity measurements in detecting and defining smallscale, isolated subsurface features.
\end{abstract}




\section{Introduction}

The use of VLF radio fields in the general context of geological mapping has been extensively reviewed by McNeill and Labson (1991). The VLF technique makes use of one or more distant radio transmitters operating between 15 and $30 \mathrm{kHz}$. The limited bandwidth means that, although several transmitters may be used at different frequencies, a main attribute of the method is that of a single-frequency EM sounding.

The lack of bandwidth is compensated for by the fact that the instrumentation is very portable and cost-effective. The VLF method was developed as an inductive survey technique measuring the amplitude and subsequently phase relationship between the vertical secondary. component of the magnetic field $(Z)$ relative to the horizontal primary magnetic field $H$. This method, referred to here as VLF-Z (elsewhere as VLF-EM), relies on wavefield interaction with two dimensional (2D) and three dimensional (3D) resistivity structure.

The technique has since been extended to include a measure of the induced horizontal electric field component $E$ perpendicular to $H$. This VLF-R measurement provides a surface impedance value (e.g., $E / H$ ), usually expressed as apparent resistivity and phase, using short (e.g., 5 to $10 \mathrm{~m}$ ) electric dipoles. The VLF-R measurement contains only marginal information on the vertical resistivity structure (Fischer et al., 1983) because, in effect, only a single frequency is available (Mathieson and Crossley, 1982). These factors suggest that the strength of the VLF method lies predominantly in the definition of lateral gradients in the subsurface.

Increasingly the method is being applied to environmental and hydrogeological problems with a growing emphasis on quantitative interpretation. This applies both to mapping applications (e.g., Guerin et al., 1994) and the determination of the 2D resistivity distribution along profiles (e.g., Beamish, 1994). The frequency bandwidth of VLF has been extended to higher-frequency transmitters (to $240 \mathrm{kHz}$ ) and the multi-frequency method is referred to as radiomagnetotellurics (Turberg et al., 1994; Zacher et al., 1996).

The main interpretational difficulty with the VLF, and radiomagnetotelluric, methods stems from the directional polarisation of the incident field (McNeill and Labson, 1991). This has profound implications for the manner in which the primary field couples with general 2D and $3 \mathrm{D}$ resistivity environments. The literature provides very little information on the practical aspects of interpretation when subsurface targets are complex and 3D. In large part this stems from the fact that such information can only be obtained through three-dimensional EM modelling. To clarify the interpretation of 'difficult' VLF data sets a 3D modelling study at VLF frequencies was undertaken.

One two-body model and two single-body models are considered. The single-body models consist of a concealed, isolated, vertical conductive feature. The lateral extent of the feature (>60 m) allows both 2D (e.g., strike definition) and 3D (e.g., end effects) aspects to be investigated. The study can be considered relevant to fault-mapping (geological context), mapping a linear contaminant feature (environmental application. or investigating an extensive, fluid-filled fracture system (hydrogeological investigation), Guerin et al. (1994) introduced the concept of rotational invariants, obtained from two or more VLF transmitters, to allow for the correction of the directional dependence. Such invariants, here extended to include the phase response, are also discussed for the 3D models considered. 


\section{VLF in a 1D environment}

In a 1D resistivity environment (no lateral resistivity gradients) the VLF-Z measurement returns a zero value for both components (in-phase and in-quadrature) of the $\mathrm{Z} / \mathrm{H}$ ratio since no secondary currents (along lateral boundaries) are induced. The VLF-R measurement always provides apparent resistivity and phase values that are essentially the same for all transmitter frequencies, i.e., over all directions of the inducing field. If the subsurface is also vertically uniform over the depth scale corresponding to the depth of investigation (see below), the apparent resistivity will be equal to the true (uniform) resistivity of the material and the phase value will be $45^{\circ}$. All transmitters used will provide essentially the same values. If the subsurface can be approximated by two layers (within the depth of investigation), then it is generally assumed that a conductor above a resistive layer provides phase angles of $<=45^{\circ}$ while a resistive layer above a conductor provides phase angles of $>=45^{\circ}$ (Arcone, 1979).

An effective penetration depth is a depth below which resistivity changes have little influence on the response measured at the surface. According to Spies (1989) a reasonable estimate for the depth of investigation of plane-wave methods is about 1.5 skin-depths. At $20 \mathrm{kHz}$, VLF depths of investigation range from $17 \mathrm{~m}$ in a conductive $(10 \Omega \mathrm{m})$ environment, through to $54 \mathrm{~m}$ in a 'typical' $100 \Omega$.m environment, and down to $121 \mathrm{~m}$ in a resistive $(500 \Omega \mathrm{m})$ environment.

\section{VLF in 2D and 3D environments}

The main interpretational difficulty with the VLF method stems from the directional polarisation of the incident field. In VLF surveys, electric and magnetic fields are always measured at right angles. The VLF magnetic receiver coil is first rotated about a vertical axis until maximum $\mathrm{H}$ signal (Hmax) is obtained. Two electrodes are then positioned perpendicular to the direction of Hmax and are used to record Ex. As noted by Fischer et al. (1983), the Ex electric field is simply measured at a right angle to the Hmax direction and no effort is made to find the direction for which this field would reach a maximum amplitude.

The directional nature of the VLF measurement has profound implications for the manner in which the primary field couples with general 2D and 3D structures and the method of interpretation. The way VLF interacts with 2D structure is discussed by Fischer et al. (1983) and McNeill and Labson (1991). The assumption of infinite strike (which defines the 2D case) provides two decoupled modes involving separate combinations of the field components. The TEmode (or E-polarisation, electric field parallel to strike) involves surface fields Ex, Hy and Hx. The TM-mode (or $\mathrm{H}$-polarisation, magnetic field parallel to strike) involves only the surface fields Hx, Ey and Ez.

The two principle modes of induction when the VLF transmitter direction is either parallel to the geological strike (TE-mode) or perpendicular to geological strike (TM-mode) are illustrated in Fig. 1. When VLF measurements can be obtained in one, or both, of these modes, interpretation can proceed in a straightforward manner, and 2D forward modelling and inversion becomes possible (Beamish, 1994). An important consideration in a 2D context is the joint information provided by VLF-R and VLF-Z measurements. As discussed above, an Hx field is absent from the TM-mode response and the Hx measurement can therefore be used as a means of 'mode-identification'. 
Interpretational difficulties are experienced when field data are collected at azimuths which do not provide either of the two principal modes and/or the subsurface structures are complex and threedimensional. The literature provides very little information on the practical aspects of interpretation when the 'common' case of non-principle modes are measured. In large part this stems from the fact that such information can only be obtained through three dimensional EM modelling. To clarify the interpretation of 'difficult' VLF data sets a 3D modelling study at VLF frequencies was undertaken. Although 3D subsurface bodies are used, 'elongate' bodies also allow approximate 2D behaviour to be observed towards the centre of the body where 'edge-effects' are minimal

\section{3D VLF modelling}

Recent advances in 3D plane-wave modelling using difference equations are described by Mackie et al. (1993). More recent advances and a description of the algorithm used here are provided by Mackie et al. (1994). The algorithm is described as a robust and efficient finite difference scheme that computes the surface fields of general 3D models using the minimum residual relaxation method. 3D model construction is via a 3D 'core' (in $x, y$ and $z$ ) with 2D extensions (e.g., in $x, z$ and $y, z$ ) at the edges of the core with both 3D core and 2D extensions underlain by a 1D 'base'. All three model elements are constructed using a staggered grid that conforms with skin-depth requirements.

For the VLF modelling carried out here a frequency of $16 \mathrm{kHz}$ was used throughout. The background material (in which anomalies are embedded) has a resistivity of $100 \Omega \mathrm{m}$. This provides a background skin-depth of $40 \mathrm{~m}$. Only conductive $(10 \Omega \mathrm{m})$ anomalies are considered here. The skin-depth within the anomalous conductive region is $12.5 \mathrm{~m}$. The central model core comprised $32 \times 32 \times 15$ blocks with the centre $100 \times 100 \mathrm{~m}$ of the core (in $x, y$ ) made up of blocks with lateral dimensions of $5 \mathrm{~m}$. The 15 blocks making up the vertical distribution were assigned initial $(0-10 \mathrm{~m})$ block thicknesses of $2.5 \mathrm{~m}$, thereafter increasing in thickness with depth. Complex surface field values were converted to apparent resistivity and phase for display.

In the following study all the bodies are electrically-thin (in either thickness or width) at VLF frequencies. Such near-surface bodies respond galvanically rather than inductively. The principle EM response perturbation in such cases occurs in amplitude (apparent resistivity) with only minor perturbations in the VLF-R phase and in the VLF-Z field. Although such field variations are too small to be readily detected by field surveys, the modelling procedure is highly accurate $(1 \%)$. The modelled EM field perturbations, although small, can be contoured to provide an accurate mapping of the response. The small perturbations are presented here as a guide to the response patterns that would be anticipated from larger scale bodies with a larger inductive response. Banded contours, which emphasise the changes in spatial gradient, are used to display the field parameters obtained.

\section{Results of 3D modelling}

\subsection{Model 1 (two bodies)}

This model was designed to illustrate the field behaviour of elongate anomalies with strike 
directions in the same (principal) directions of VLF transmitters. The study approximates a 2D case, providing 'ideal' TE- and TM-mode behaviour with end-effects added due to the finite strike length of the bodies. The two bodies are directed $\mathrm{E}-\mathrm{W}(x$-axis)

and $\mathrm{N}-\mathrm{S}$ ( $y$-axis) and are identical apart from orientation. Each has a length of $40 \mathrm{~m}$, a width of $5 \mathrm{~m}$ and a thickness of only $5 \mathrm{~m}$. The bodies are 'concealed' and extend from 2.5 to $7.5 \mathrm{~m}$ in depth. Since the bodies are 'electrically thin' and shallow the secondary fields generated will be largely galvanic, i.e., the main anomaly will be generated in amplitude (apparent resistivity). Body $1(\mathrm{E}-\mathrm{W})$ extends from $(-40,-25.25 \mathrm{~m})$ to $(0,25.25 \mathrm{~m})$ and body $2(\mathrm{~N}-\mathrm{S})$ extends from $(25.25,0$ $\mathrm{m})$ to $(25.25,40 \mathrm{~m})$.

Apparent resistivity and phase behaviour is shown for $E$-polarisation (transmitter E-field polarised $\mathrm{E}-\mathrm{W}$. in Fig. 2. In this polarisation, Body $1(\mathrm{E}-\mathrm{W})$ responds in a TE-mode and Body $2(\mathrm{~N}-\mathrm{S})$ in a TMmode. In Fig. 2, Body 1 is responding in the TE-mode. A N-S profile across the centre of the body reveals $2 \mathrm{D}$ type behaviour with a central minimum of $70 \Omega \mathrm{m}$ 'outlining' the anomaly. Through the central region, the apparent resistivity returns slowly to its background value. The finite length of the body is revealed by a concentric increase in apparent resistivity at the body ends. Body 2 is responding largely in the TM-mode (Ex current flow is perpendicular to the $\mathrm{N}-\mathrm{S}$ axis of the body). A smaller amplitude anomaly with a much decreased 'half-width' is observed. The associated phase variations, also shown in Fig. 2, comprise a variation of only $4^{\circ}$ since the bodies are responding galvanically. It is arguable whether such variations could be detected in a field survey using an instrument with a phase resolution of the order of $1^{\circ}$. Despite the small level of the anomaly, the general pattern of phase behaviour is similar to that of the amplitude variations.

Again since the response is largely galvanic, the VLF-Z field ratios shown in Fig. 3 are extremely small $(\sim 0.5 \%)$ and could not be detected by a field survey. Despite their small magnitude, the results demonstrate the existence of an $\mathrm{Hz}$ field for Body 1 responding in a TE-mode ( $E x$ current flow parallel to strike) and the lack of an $\mathrm{Hz}$ field from Body 2 responding in the TM-mode (Ex current flow perpendicular to strike). Small amplitude 3D edge-effects from Body 2 are also apparent. The modelling results obtained using the orthogonal polarisation (Ey-polarisation) are a simple transposition of those shown with Body $1(\mathrm{E}-\mathrm{W})$ responding in a TM-mode and Body $2(\mathrm{~N}-$ $\mathrm{S})$ responding in a TE-mode sense.

\subsection{Model 2 (single body)}

This model was designed to illustrate the field behaviour of a single elongate anomaly whose strike axis is rotated by 17 and $73^{\circ}$ from the principal directions. The conductive body has a strike length of about $62 \mathrm{~m}(-30$ to $+30 \mathrm{~m}$ along the $y$-axis), a width between 5 and $10 \mathrm{~m}$ and is concealed. The body extends from 2.5 to $20 \mathrm{~m}$ in depth. The $(x, y)$-coordinates of the centre the body end points are $(-7.25,-30 \mathrm{~m})$ and $(7.25,30 \mathrm{~m})$.

The VLF-R results shown in the Ey -polarisation of Fig. 4 represent a $17^{\circ}$ rotation of strike from the direction of induction (transmitter N-S), i.e., the TE-mode. The anomaly pattern is similar to that observed for the TE-mode response of Body 1 in the previous example (Fig. 2). Here the central minimum contour of $60 \Omega \mathrm{m}$ provides an outline of the concealed anomaly with the correct strike. End effects provide a localised increase in apparent resistivity to $120 \Omega \mathrm{m}$. Phase angles for Ey polarisation are defined in the second quadrant $\left(90\right.$ to $\left.180^{\circ}\right)$. The phase response is again small due to the electrically-thin nature of the body which results in a largely galvanic response. The gradient anomaly pattern does, however, indicate that the true strike of the body is less well-identified in the VLF-R phase response. Again, since the response is largely galvanic, the VLF-Z field ratios shown in Fig. 5 are small. The central gradients generated, however, do indicate the correct strike orientation of the conductor. 
The Ex -polarisation results for Model 2 define an anomaly strike direction that is $17^{\circ}$ from the TMmode. The VLF-R results are shown in Fig. 6 . The anomaly pattern is similar to that observed for the TM-mode response of Body 2 in the previous example (Fig. 2). In the present case, the central minimum contour of $50 \Omega \mathrm{m}$ outlines the concealed anomaly. The gradient anomaly pattern in phase provides a central 'low' that is closely associated with the strike of the body. The anomaly rotation away from a true TM-mode provides a VLF-Z response as shown in Fig. 7. The $Z / H$ amplitudes are reduced by a factor of 2 from the previous case (Fig. 5). The strike of the anomaly is not well-defined.

\subsection{Model 3 (single body)}

This model is designed to illustrate the field behaviour of a single elongate anomaly whose strike axis is rotated by 34 and $56^{\circ}$ from the principal VLF directions. The conductive body has a central strike length of $67 \mathrm{~m} \mathrm{(-30} \mathrm{to} 30 \mathrm{~m})$ along the $y$-axis. and a width of between 5 and $10 \mathrm{~m}$. The body is again concealed and extends from 2.5 to $20 \mathrm{~m}$ in depth. The $(x, y)$-coordinates of the centre body end points are $(-15,-30 \mathrm{~m})$ and $(15,30 \mathrm{~m})$. The VLF-R results shown in the Ey-polarisation of Fig. 8 represent a $34^{\circ}$ rotation of strike from a VLF transmitter due N-S, i.e., a $34^{\circ}$ rotation from a TE-mode response. The results obtained indicate that the $3 \mathrm{D}$ response attributes, in both amplitude and phase, map the body ends very well. In the absence of such terminations (e.g., a 2D case) the results indicate that the strike would be overestimated by the central resistivity low and would be poorly defined in the phase response. The corresponding VLF-Z results shown in Fig. 9 are surprising. The anomaly pattern generated by a $34^{\circ}$ rotation from the principal TE-mode direction appears to map the strike direction adequately. The VLF-Z anomaly is generated by excess currents induced along the resistivity gradients provided by the body. It appears that even with a strike deviation of $34^{\circ}$ from the principal TE-mode, sufficient TE-mode behaviour is generated to map the anomalous structure.

The $E x$-polarisation results for Model 3 define an anomaly strike direction that is $34^{\circ}$ from the principal TM-mode. The VLF-R results are shown in Fig. 10. In this case the central minimum contour of $40 \mathrm{~V}$ m outlines the concealed body while the associated phase low of $43^{\circ}$ appears to underestimate the strike direction. Complex patterns of high values (in both amplitude and phase) are generated by the $3 \mathrm{D}$ ends of the body. The response being considered can also be understood as a $56^{\circ}$ rotation from the TE-mode direction which generates the VLF-Z field. The VLF-Z response is shown in Fig. 11. As with the corresponding case of Model 2 (Fig. 7), the strike of the anomaly is poorly defined and the amplitudes of the VLF-Z response have been further reduced.

\section{Use of rotational invariants to map structure}

In a recent paper (Guerin et al., 1994), the use of invariants for correcting VLF field polarisation effects is discussed. The use of invariants follows from the standard procedures of tensor magnetotellurics (MT) in which four components ( $E x, E y, H x$ and $H y)$ of the horizontal fields are measured and a tensor impedance $(Z)$ relationship of the form:

$$
\left[\begin{array}{l}
E_{x} \\
E_{y}
\end{array}\right]=\left[\begin{array}{ll}
Z_{x x} & Z_{x y} \\
Z_{y x} & Z_{y y}
\end{array}\right]\left[\begin{array}{l}
H_{x} \\
H_{y}
\end{array}\right]
$$

is used. In the VLF method, $E x$ and Ey are provided by two different transmitters, and the two measurements can only provide the off-diagonal components $Z x y$ and $Z y x$. This is because, in the general case, three simultaneous measurements (one $E$ and two $H$ 's) are required to determine the coupled tensor elements and VLF conventionally only performs two such measurements (one $E$ and one $H$ ). 
In MT the use of rotationally invariant parameters of the impedance tensor has been suggested in an attempt to minimise multidimensional effects. Berdichevsky and Dmitriev (1976) proposed two different rotationally-invariant averages of the tensor as $Z_{\mathrm{av}}=(Z x y-Z y x) / 2$ where the subscript denotes an average and a determinant average: $Z_{\text {deta }}=\left(Z_{x x} Z_{y} y-Z_{x} \text { } Z_{y}\right)^{1 / 2}$. A simplified invariant, $Z_{\text {det }}=(-$ $Z x y Z y x)^{1 / 2}$ with ZxxZyy $<<Z x y Z y x$ is more suitable for VLF applications.

All invariants are independent of the measurement directions. In our model studies all three invariants have been found to give very similar results and we here concentrate on the use of the simplified determinant average $Z_{\text {det. }}$ Guerin et al. (1994) discuss invariants only in terms of apparent resistivity, however, the invariant phase is also useful. In terms of the measured VLF quantities of apparent resistivity $(\rho)$ and phase $(\varphi)$ we have $\rho_{\operatorname{det}}=(\rho x y \text {. } \rho y x)^{1 / 2}$ and $\varphi_{\operatorname{det}}$ $=\varphi x y+\varphi y x$.

If both $\varphi x y$ and $\varphi y x$ are defined in the first quadrant $\left(0\right.$ to $\left.90^{\circ}\right)$, then $\varphi_{\text {det }}$ is defined in the range 0 to $180^{\circ}$ with a value of $90^{\circ}$ representing a uniform half-space response. The ability of $\rho_{\text {det and }} \varphi_{\text {det }}$ to map structure provided by the three test models is now considered. The two polarisations (Ex and Ey) used to obtain the invariant represent stimulation of the models by two orthogonal VLF transmitters.

\subsection{Model 1 (two bodies)}

This model is characterised by two elongate anomalies with strike directions in the same (principal) directions as the two VLF transmitters. In this 'simple' situation, the invariant response, as shown in Fig. 12, provides an 'ideal' mapping of the anomalies associated with both conductive bodies. Both anomalies have the same half-width in amplitude and the four ends (of the two bodies) are identified by a similar increase in phase (above $90^{\circ}$ ) and an associated high (above $90^{\circ}$ ) outlining each body.

\subsection{Model 2 (single body)}

This model is characterised by a single body with a strike rotated by 17 and $73^{\circ}$ from the principal directions provided by the two VLF transmitters. In this case the invariant response, shown in Fig. 13, provides a relatively simple mapping of the true strike and extent of the body. The interpretation of the invariant is more straightforward than an assessment of the individual maps provided by the two transmitters. The invariant phase in particular 'simplifies' to a low value $\left(<90^{\circ}\right)$ above the body with an increase $\left(>90^{\circ}\right)$ defining the terminating ends.

\subsection{Model 3 (single body)}

This model is characterised by a single body with a strike rotated by 34 and $56^{\circ}$ from the principal directions provided by the two VLF transmitters. In this case the invariant response, shown in Fig. 14, again provides a straightforward mapping of true strike and extent of the body. A very similar anomaly mapping to the previous case (Model 2, Fig. 13) occurs in both amplitude and phase.

\section{Summary of 3D modelling results}

The main conclusions of this study concern the mapping capability of directional and invariant VLF data. For 2D modelling/inversion, the field distortions introduced by directional complexity remain important. The first model considered the case of a VLF transmitter providing a principal (TE or TM) mode of induction. In this case the VLF-R results provide anomaly mapping in both TE (current parallel to strike) and TM (current perpendicular to strike) modes. The TM-mode response produces a spatially more compact anomaly than that of the TE-mode. The use of VLF-R invariant mapping, obtained from two orthogonal transmitters, simplified interpretation as shown in Fig. 12. 
The invariant amplitude displays an absence of 3D end-effects while the invariant phase moves to high values in response to body termination points. A VLF-Z response only occurs in the TE-mode response. For the model studied, 3D contributions to the VLF-Z response are minor.

The second two models examined body strike rotations of 17 and $34^{\circ}$ from both principal directions (i.e., VLF transmitters to the south and west). For both strike rotations and for both modes similar conclusions apply to the VLF-R response. The apparent resistivity mapping appears to approximately identify the 2D strike of the body. The phase response is more complicated and, for the models studied, appears to be dominated by 3D body end-effects. Clearly for the smaller rotation of $17^{\circ}$ the results obtained are 'closer' to the case of 'principal direction' behaviour (e.g., Model 1). For the rotations considered, the use of invariant mapping provides an important simplification for interpretation purposes. Again the invariant amplitude displays an absence of 3D end-effects and the phase 'identifies' the body termination points. Overall the invariant is seen to provide correct strike and body extent information.

It is noteworthy that the VLF-Z response for the rotation angles of 17 and $34^{\circ}$ from principal TE-mode identifies the correct body strike. Additional studies confirm that this behaviour is repeated for the rotation range from 0 to $45^{\circ}$ from the principal TE-mode direction. With increasing rotation angle, the magnitude of the VLF-Z anomaly decreases. The VLF-Z response behaviour for rotations above $45^{\circ}$ from TE-mode is illustrated by the rotations of 17 and $34^{\circ}$ from the principal TMmode direction. These results also relate to 73 and $54^{\circ}$ rotations from the principal TE-mode. In both of these cases the magnitude of the VLF-Z response rivals that of the previous case but the body strike direction is inaccurate.

Finally, it is worth noting that the type of anomalies considered here are electrically-thin and compact. Such anomalies respond, even at VLF frequencies, largely in a galvanic mode. Assuming an instrument resolution of order $1 \Omega \mathrm{m}$ in apparent resistivity and $1^{\circ}$ in phase, then it is clear from the results presented that such features are only detectable using measurements of apparent resistivity. Such features would be associated with largely uniform variations in phase and near-zero VLF-Z response.

\section{Acknowledgements}

The research was funded in part by the Commission of the European Communities (EC Contract No. 7220-AFr136). This study would not have been possible without the excellent 3D modelling code supplied by Randy Mackie. The report is published with the approval of the Director, British Geological Survey (NERC).

\section{References}

Arcone, S.A., 1979. Resolution studies in airborne resistivity surveying with VLF. Geophysics 44, 937946.

Beamish, D., 1994. Two-dimensional regularised inversion of VLF data. J. Appl. Geophys. 32, 357374.

Berdichevsky, M.N., Dmitriev, V.I., 1976. Basic principles of interpretation of magnetotelluric curves. In: Adam

(Ed), Geoelectric and Geothermal Studies. Akad. Kaido, Budapest, KAPG Geophys. Mono., pp. 165221.

Fischer, G., Le Quang, B.V., Muller, I., 1983. VLF ground surveys, a powerful tool for the study of shallow two dimensional 
structures. Geophys. Prosp. 31, 977-991.

Guerin, R., Tabbagh, A., Benderitter, Y., Andrieux, P., 1994. Invariants for correcting field polarisation effects

in MT-VLF resistivity mapping. J. Appl. Geophys. 32, 375-383.

Mackie, R.L., Madden, T.R., Wannamaker, P.E., 1993. Three-dimensional magnetotelluric modeling using difference

equations-theory and comparisons to integral equation solutions. Geophysics 58, 215-226.

Mackie, R.L., Smith, J.T., Madden, T.R., 1994. Three-dimensional electromagnetic modeling using finite difference

equations: the magnetotelluric example. Rad. Sci. 29, 923-935.

Mathieson, C., Crossley, D.J., 1982. Interpretation of single frequency VLF data. In: Collett, L.S., Jenson, O.G.

(Eds.), Geophysical Applications of Surface Wave Impedance Measurements. Geol. Surv. Can., Pap. 81- 15, 49-65.

McNeill, J.D., Labson, V.F., 1991. Geological mapping using VLF radio fields. In: Nabighian, M.N. (Ed.), Electromagnetic Methods in Applied Geophysics, Part B: Application. SEG, Tulsa, pp. 521-640.

Spies, B.R., 1989. Depth of investigation in electromagnetic sounding methods. Geophysics 54, 872888.

Turberg, P., Muller, I., Flury, F., 1994. Hydrogeological investigations of porous environments by radiomagnetotelluric-resistivity. J. Appl. Geophys. 31, 133-143.

Zacher, G., Tezkan, B., Neubauer, F.M., Hordt, A., Muller, I., 1996. Radiomagnetotellurics: a powerful tool for waste-site exploration. Eur. J. Environ. Eng. Geophys. 1, 139-159. 


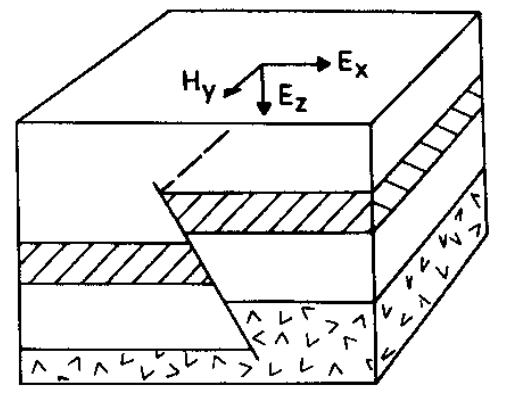

TM-Mode

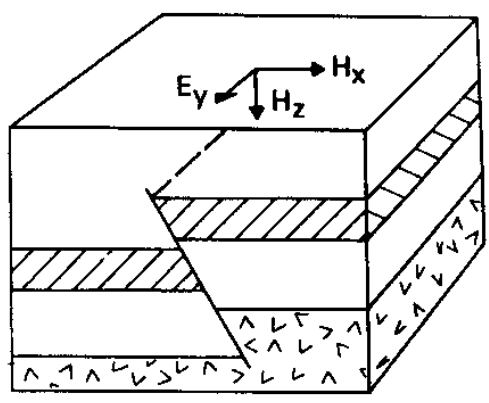

TE-Mode

Fig. 1. Principal modes of the VLF field in a two-dimensional environment. TM-mode, magnetic field parallel to strike. TE-mode, electric field parallel to strike. 

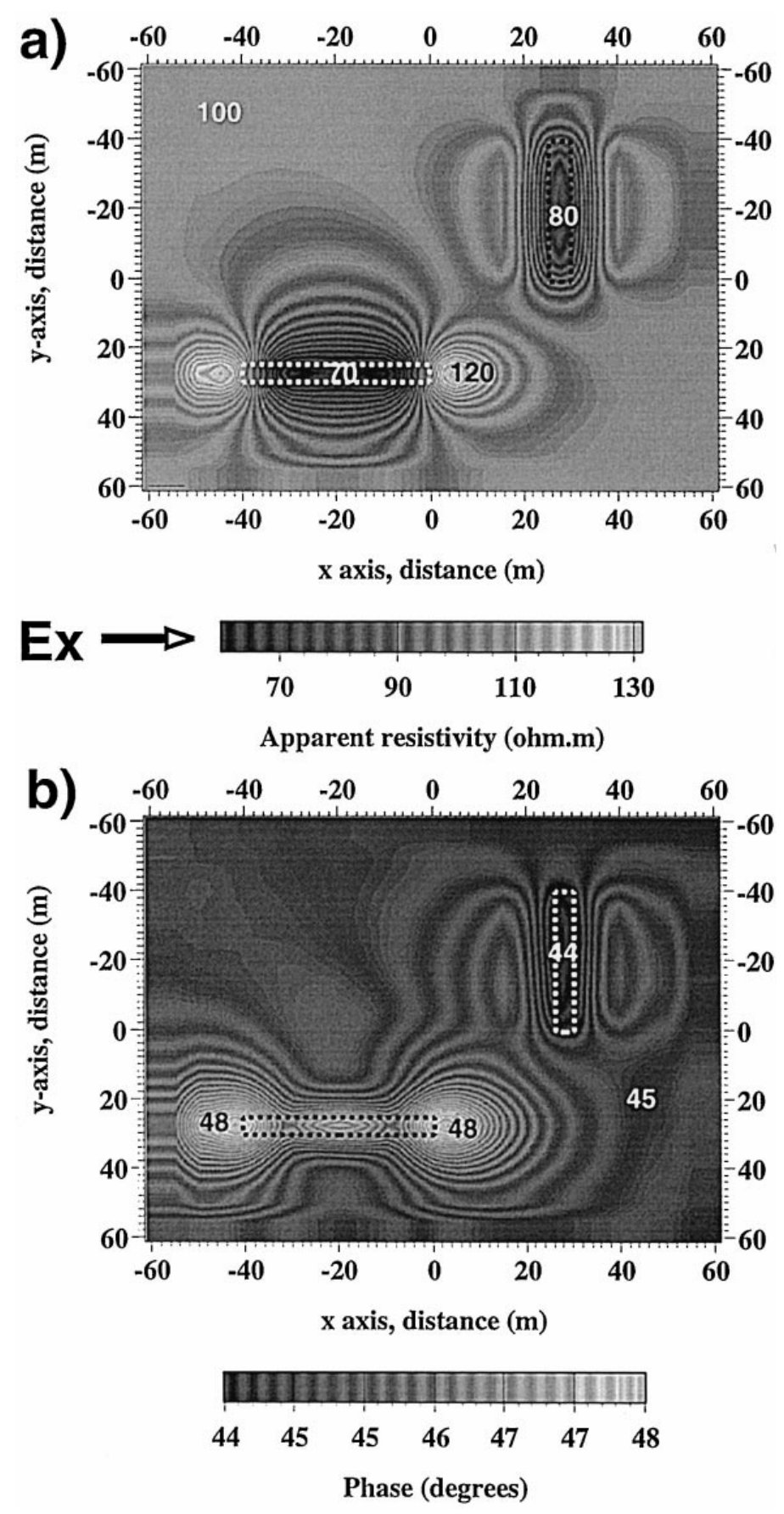

Fig. 2. Model 1 _two bodies. VLF-R results. (a) Apparent resistivity and (b) phase at $16 \mathrm{kHz}$. Ex polarisation (arrow). Dash lines denote bodies (Body 1 lowermost). 

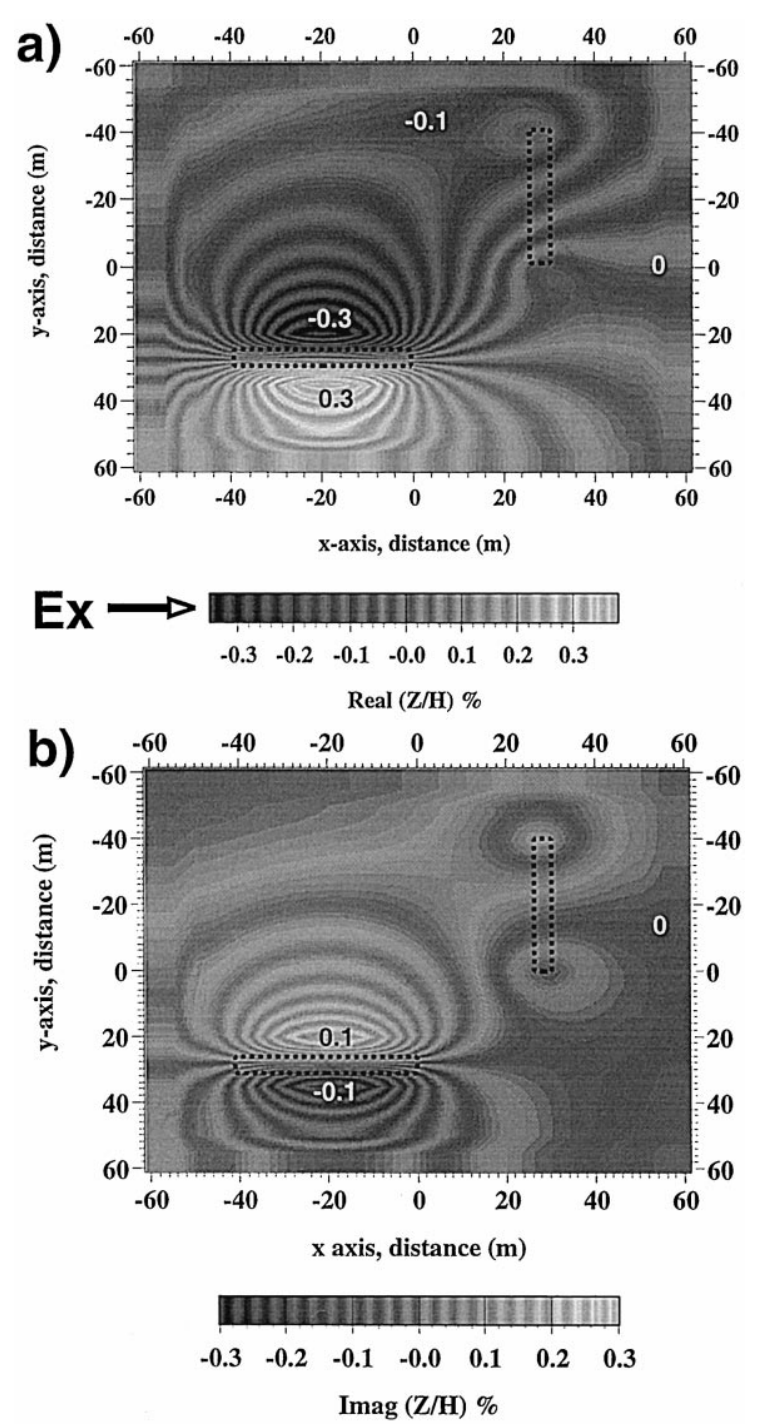

Fig. 3. Model 1 (two bodies) VLF-Z results. (a) Real Z/H. and (b) imag_Z/H. at $16 \mathrm{kHz}$. Ex polarisation (arrow). Dash lines denote bodies (Body 1 lowermost). 

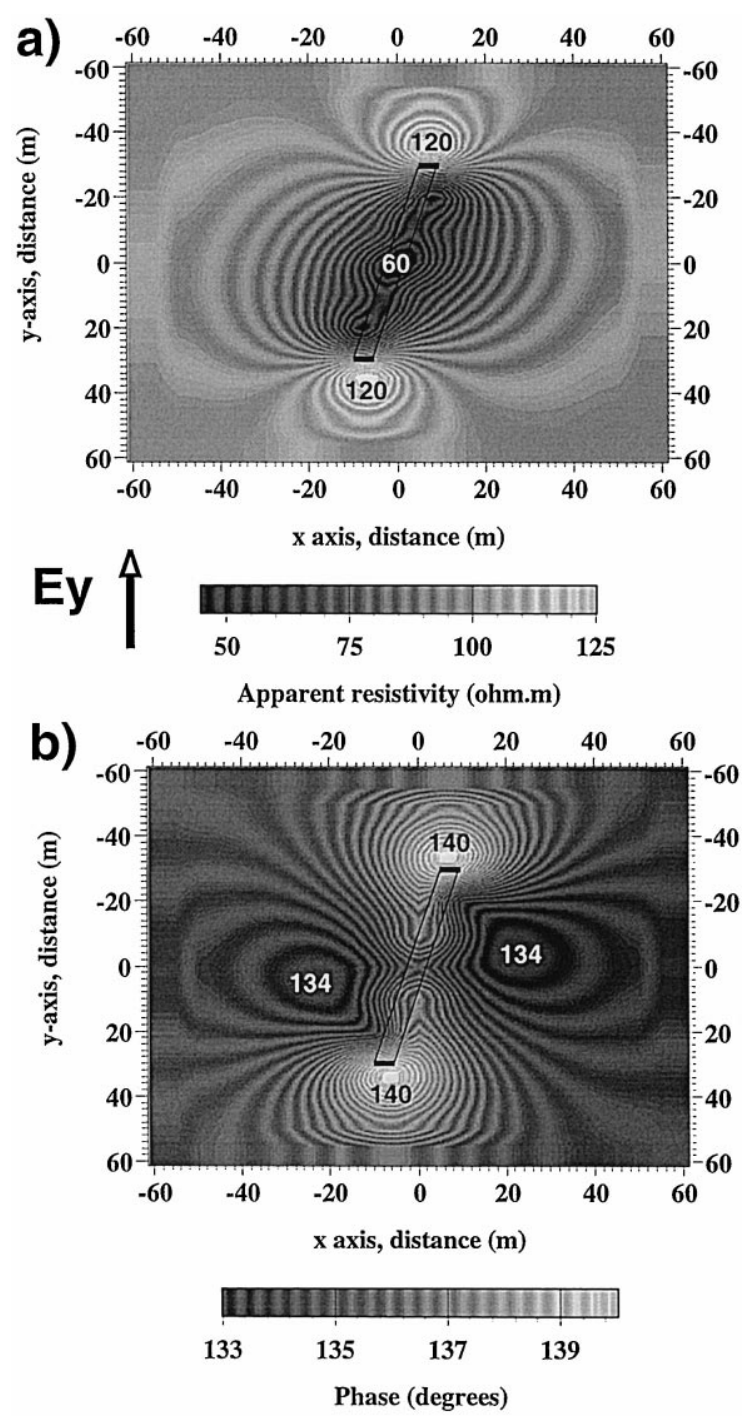

Fig. 4. Model 2 (single body) VLF-R results. (a) Apparent resistivity and (b) phase at $16 \mathrm{kHz}$. Ey polarisation (arrow). Heavy lines denote body ends. 

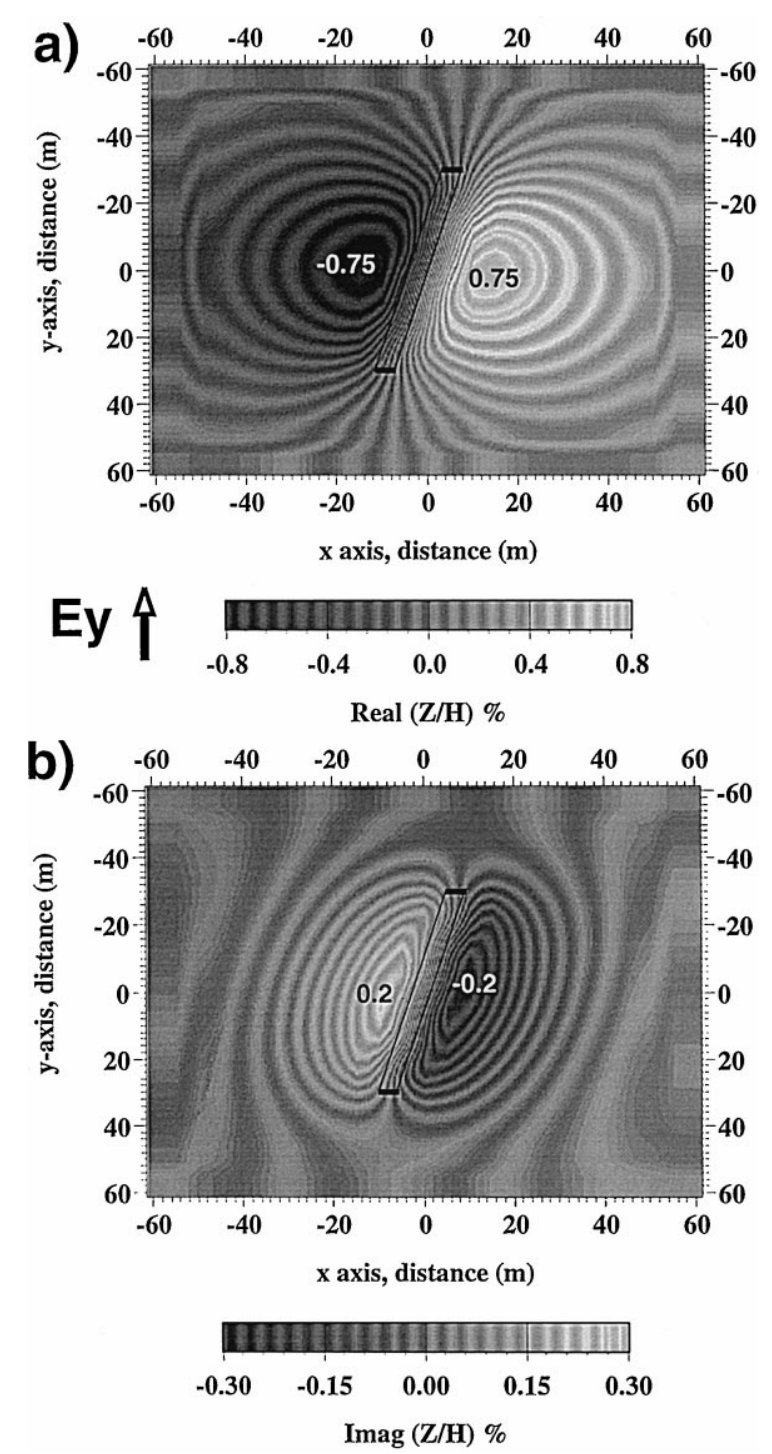

Fig. 5. Model 2 (single body) VLF-Z results. (a) Real $Z / H$ and (b) imag_Z/H. at $16 \mathrm{kHz}$. Eypolarisation (arrow). Heavy lines denote body ends. 

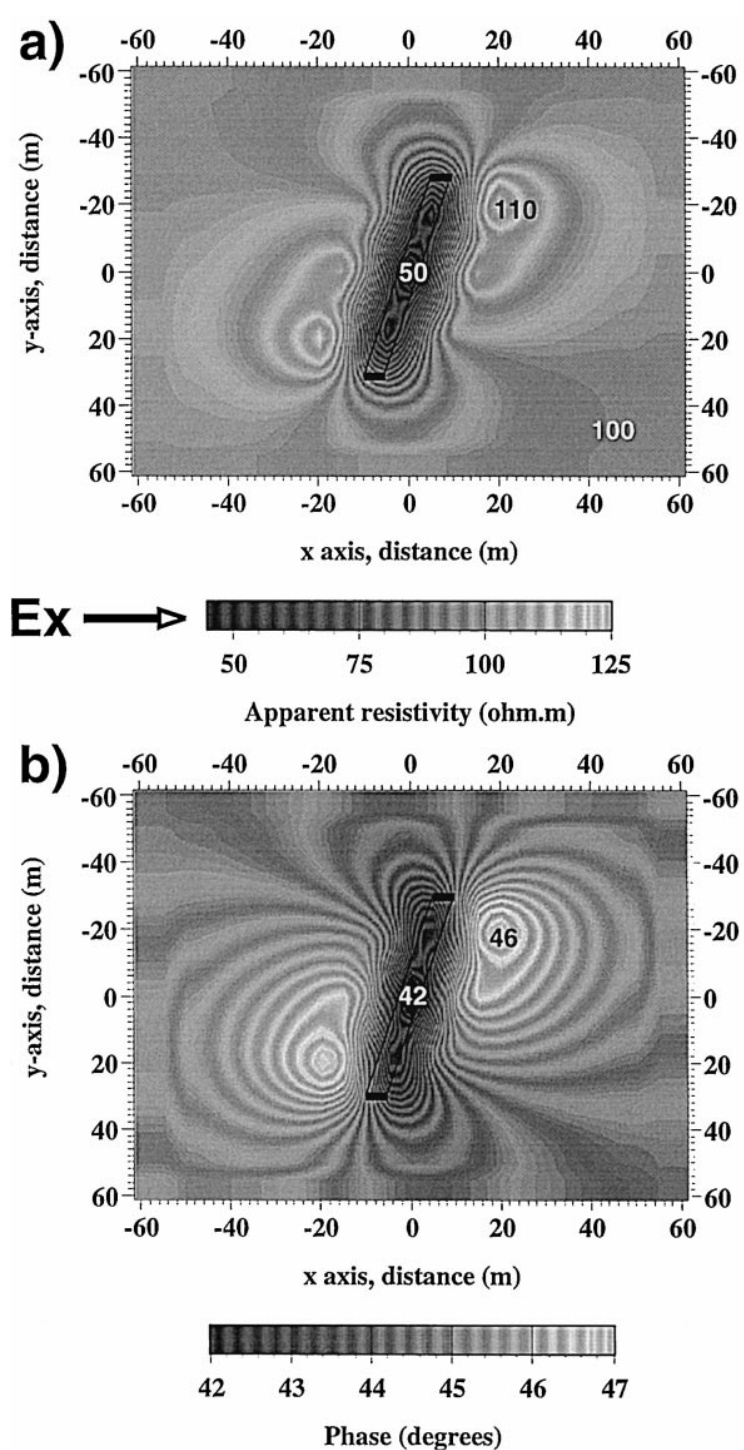

Fig. 6. Model 2 (single body) VLF-R results. (a) Apparent resistivity and (b) phase at $16 \mathrm{kHz}$. Expolarisation (arrow). Heavy lines denote body ends. 

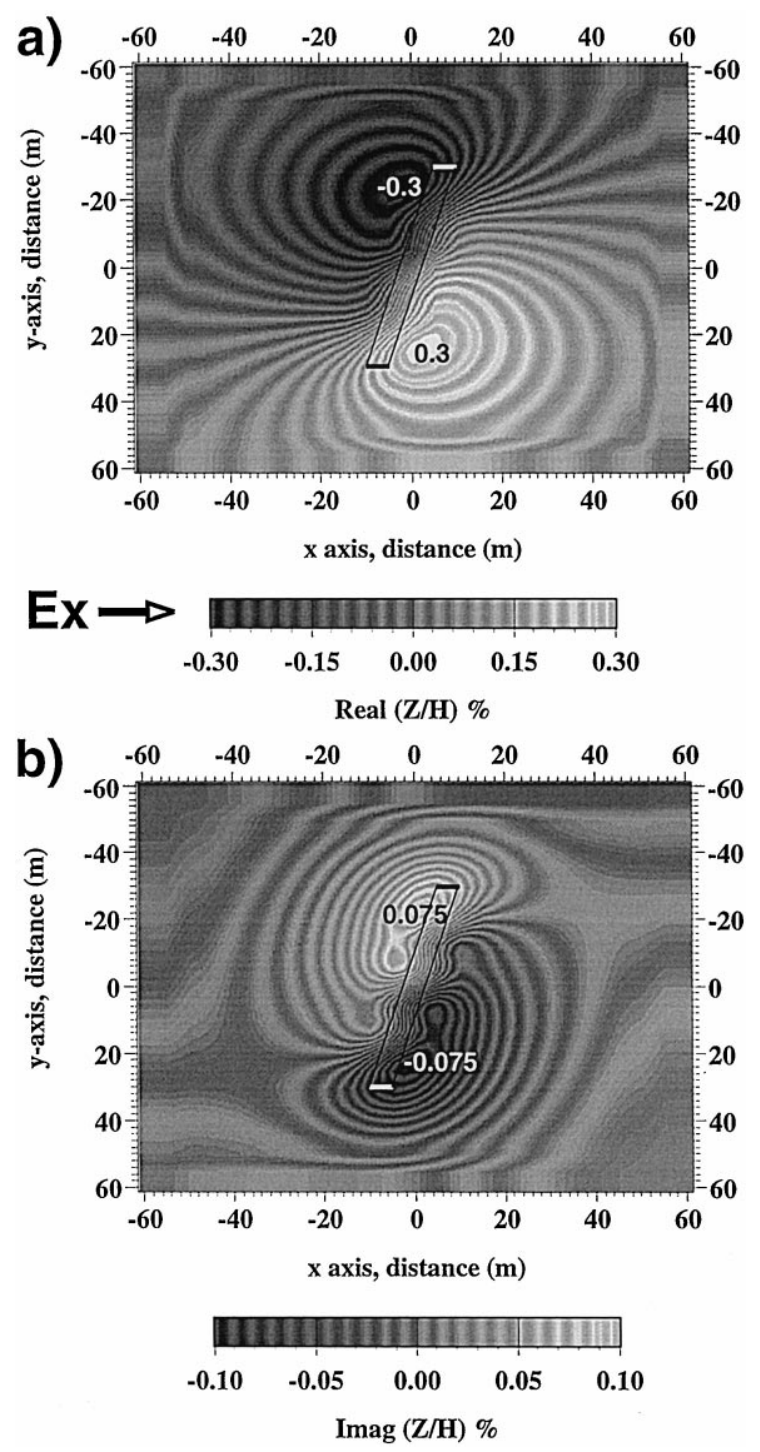

Fig. 7. Model 2 (single body) VLF-Z results. (a) Real $Z / H$. and (b) imag $Z / H$ at $16 \mathrm{kHz}$. Ex polarisation (arrow). Heavy lines denote body ends. 

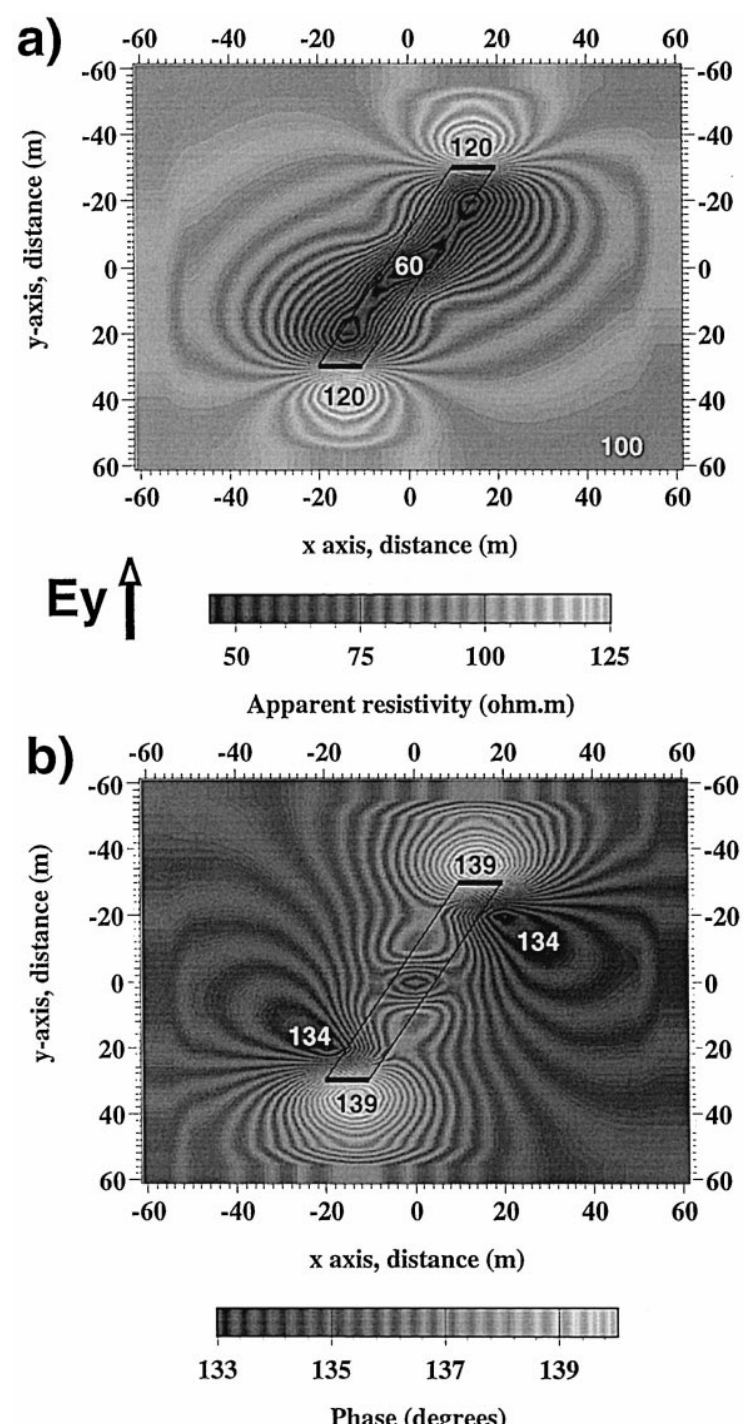

Fig. 8. Model 3 (single body) VLF-R results. (a) Apparent resistivity and (b) phase at $16 \mathrm{kHz}$. Eypolarisation (arrow). Heavy lines denote body ends. 

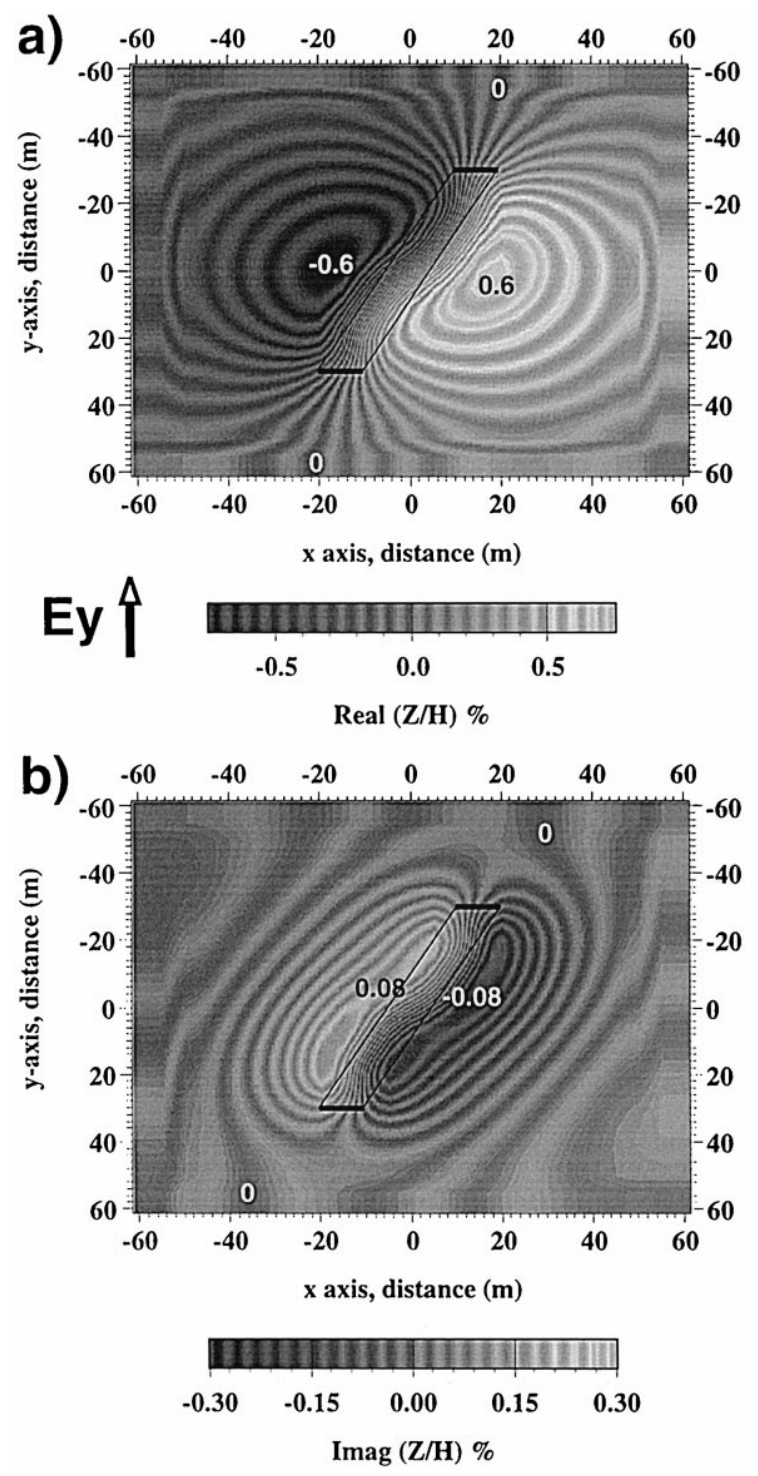

Fig. 9. Model 3 (single body) VLF-Z results. (a) Real $Z r / H$. and (b) imag $Z / H$. at $16 \mathrm{kHz}$. Ey. polarisation (arrow). Heavy lines denote body ends. 

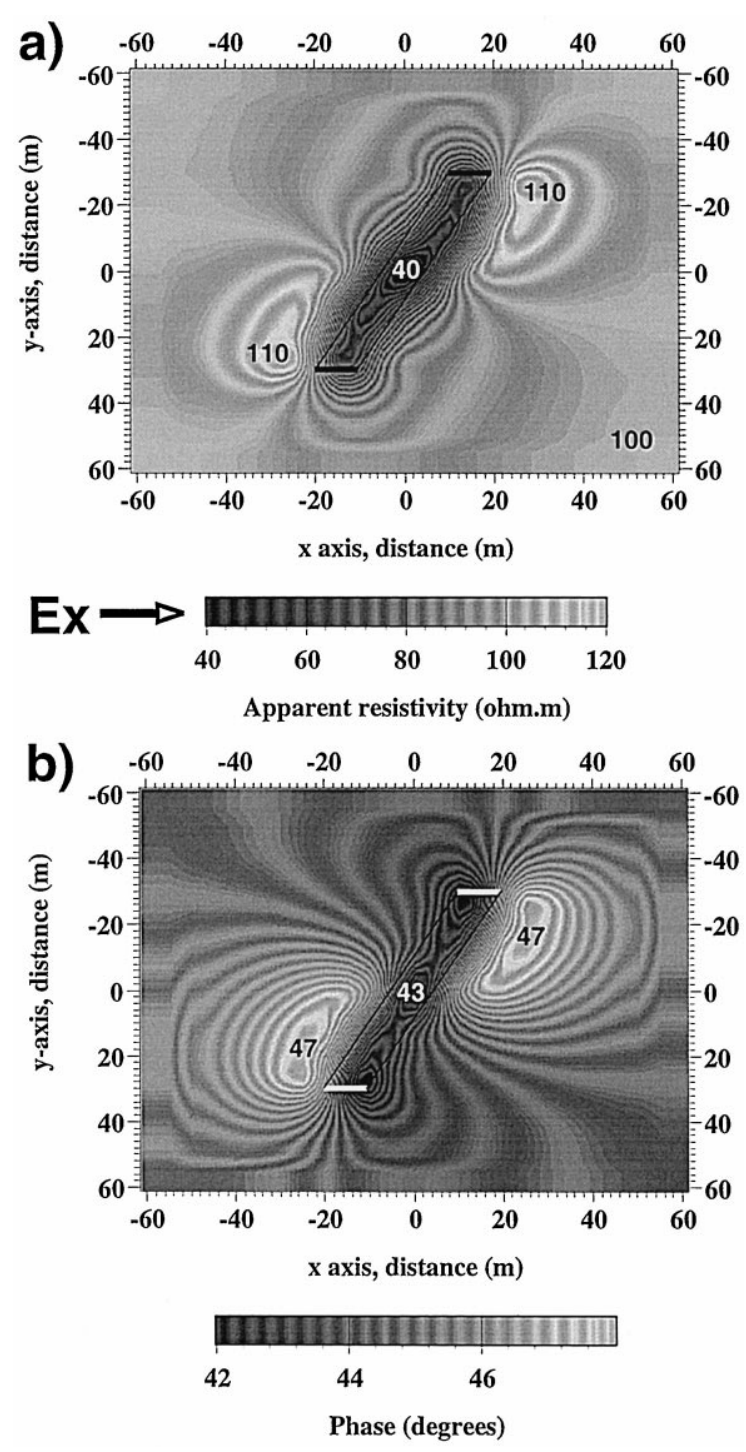

Fig. 10. Model 3 (single body) VLF-R results. (a) Apparent resistivity and (b) phase at $16 \mathrm{kHz}$. Expolarisation (arrow). Heavy lines denote body ends. 

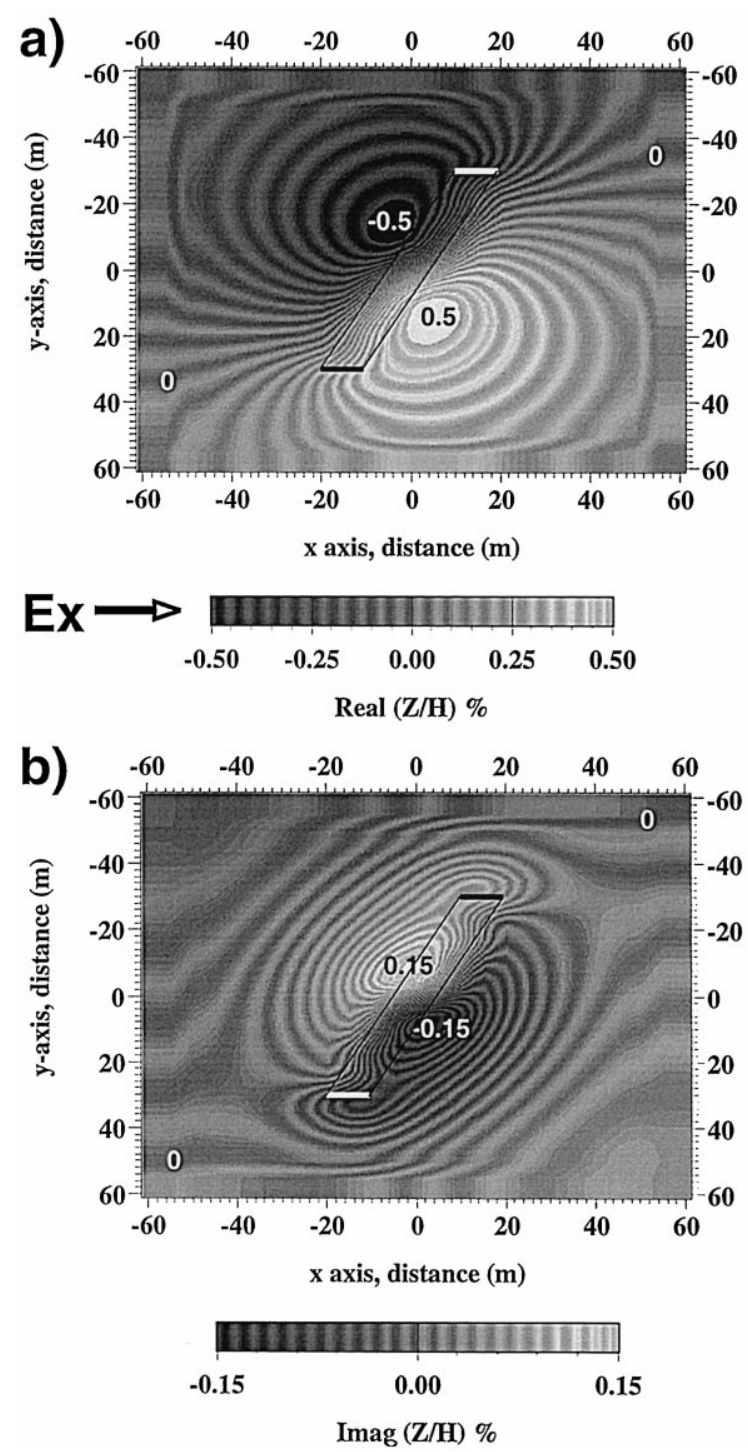

Fig. 11. Model 3 (single body) VLF-Z results. (a) Real $Z / H$. and (b) imag $Z / H$. at $16 \mathrm{kHz}$. Ex polarisation (arrow). Heavy lines denote body ends. 

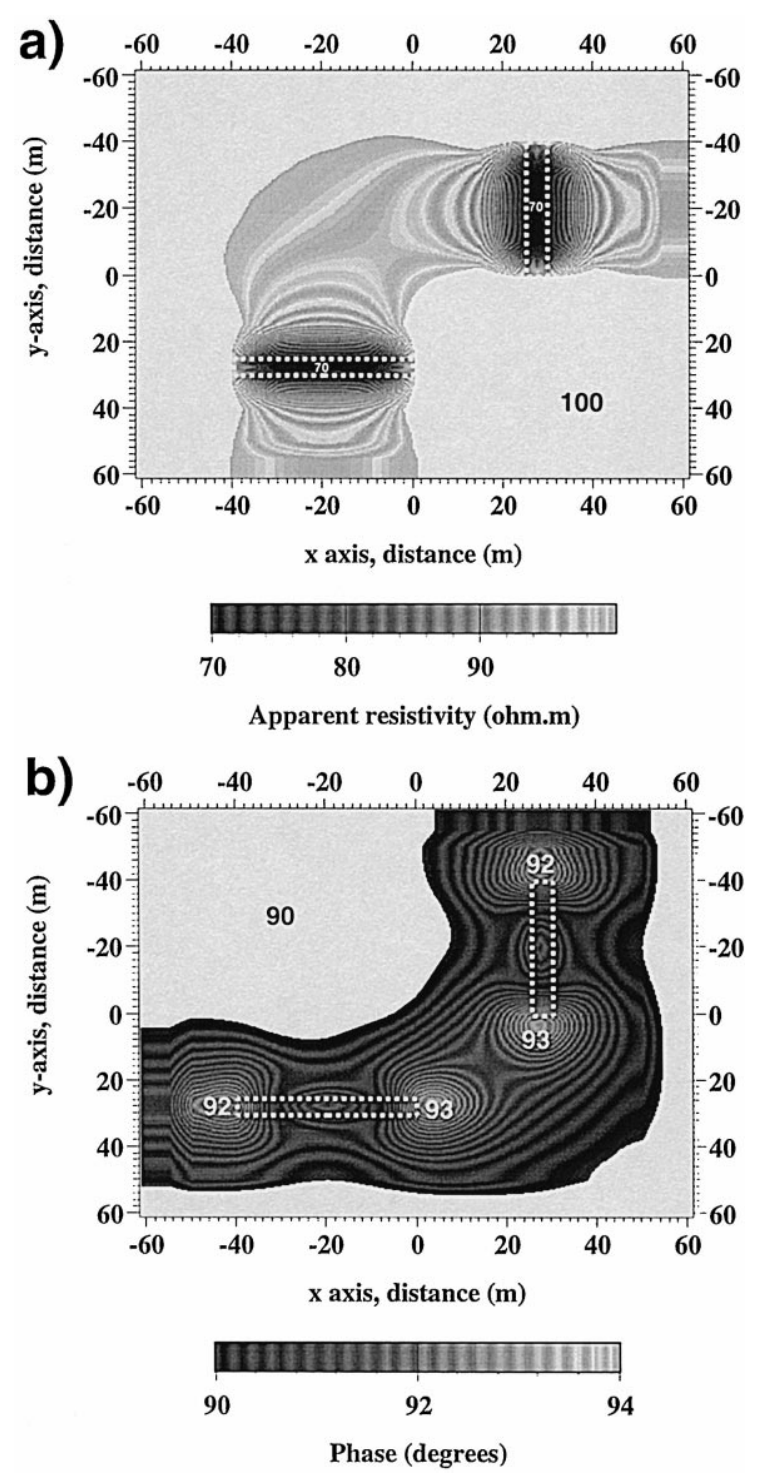

Fig. 12. Model 1 (wo bodies). invariant (determinant average) results. (a) Apparent resistivity and (b) phase at $16 \mathrm{kHz}$. Dash lines denote bodies (Body 1 lowermost). 

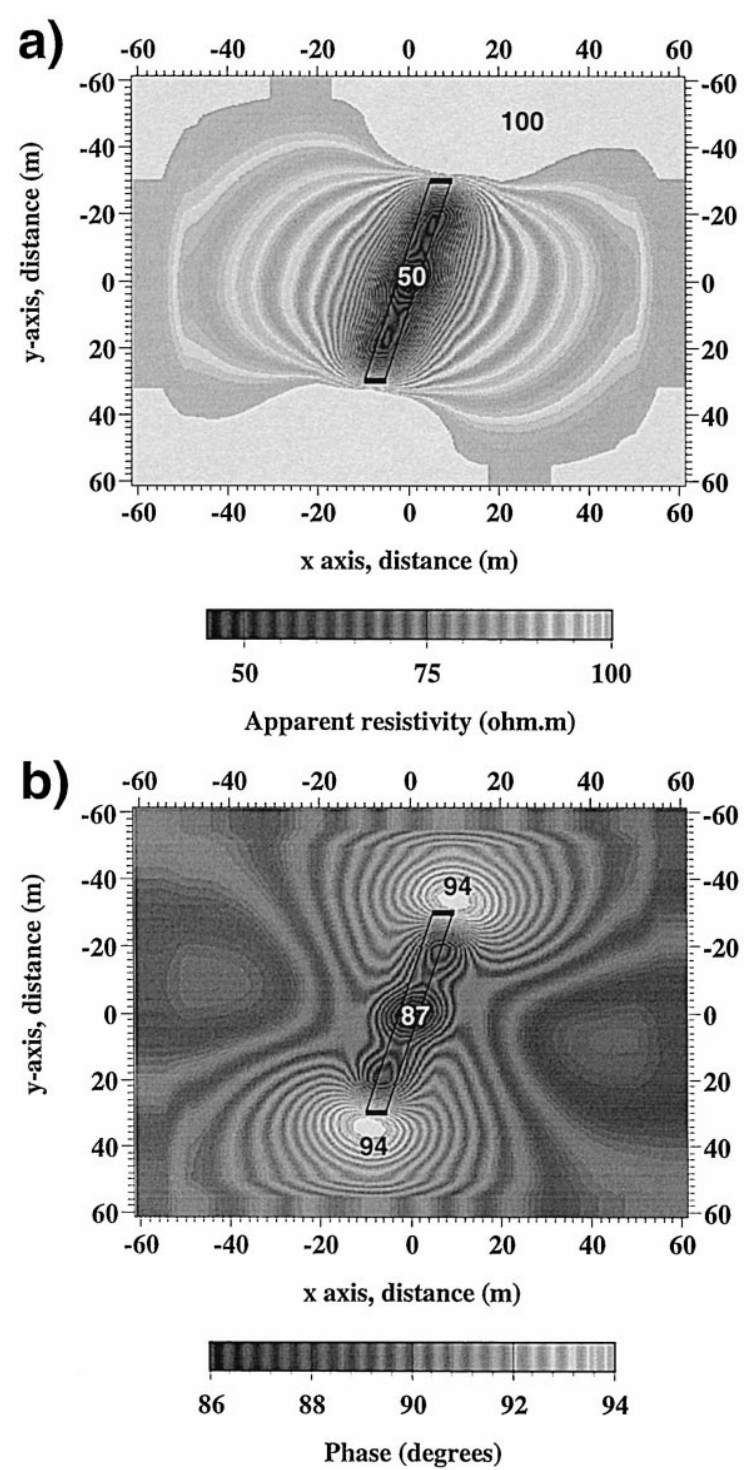

Fig. 13. Model 2 (single body) invariant (determinant average) results. (a) Apparent resistivity and (b) phase at $16 \mathrm{kHz}$. Heavy lines denote body ends. 

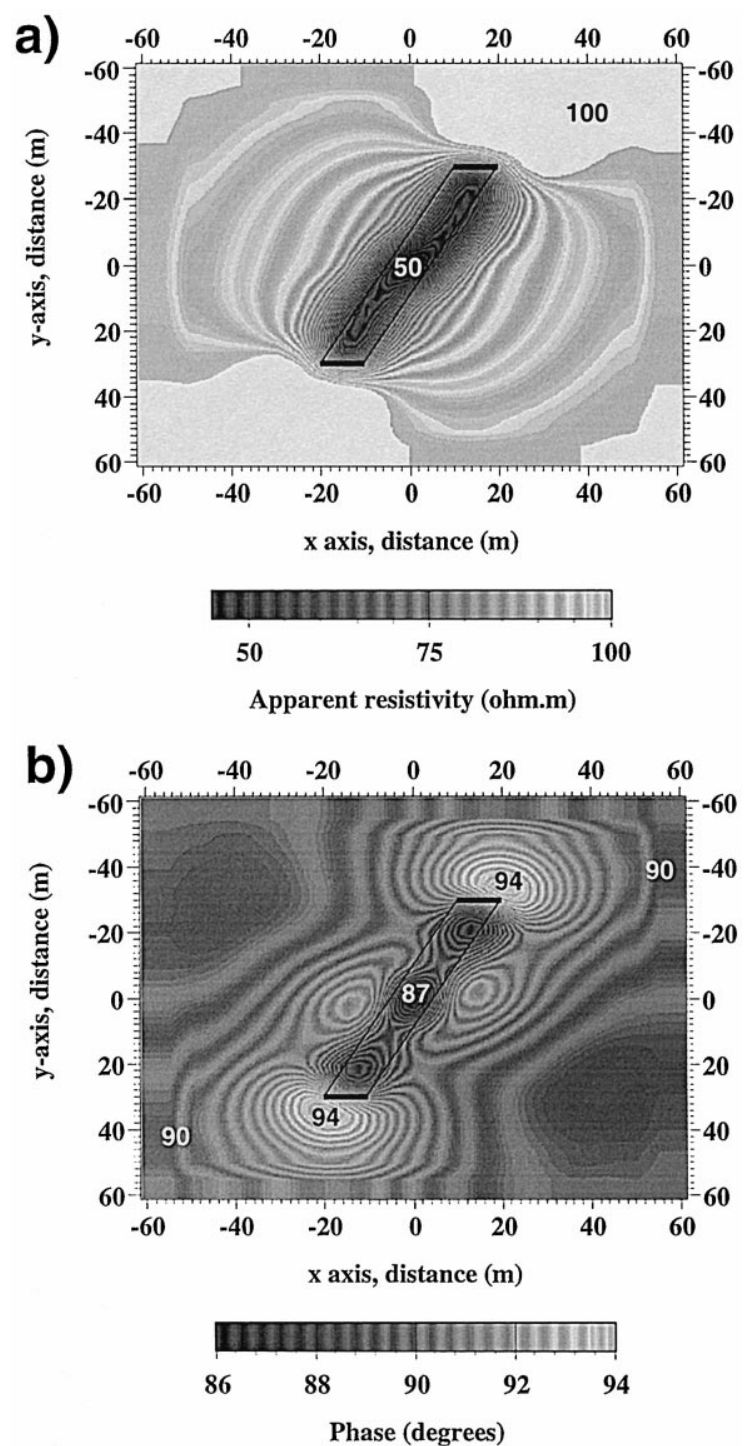

Fig. 14. Model 3 (single body) invariant (determinant average) results. (a) Apparent resistivity and (b) phase at $16 \mathrm{kHz}$. Heavy lines denote body ends. 